\title{
Postoperative cognitive dysfunction: A case report and literature review
}

\author{
Gurpreet Singh, Margaret M Cinicola, Naveen R Parva, John T Cinicola, Venkat G Muvva, Vinod K Nookala \\ UPMC Pinnacle, Harrisburg, PA, USA
}

Received: August 9, 2017

Accepted: October 16, 2017

Online Published: October 20, 2017

DOI: $10.5430 /$ crim.v4n $4 \mathrm{p} 37$

URL: https://doi.org/10.5430/crim.v4n4p37

\begin{abstract}
Background: Postoperative cognitive dysfunction (POCD) is loss of cognition especially in memory and executive function that can extend from a few days to a few weeks after surgery. It is more common in older adult patients. We present a case featuring a typical progression of POCD along with its associated preoperative risk factors.

Case report: A 65-year-old male patient with an extensive past medical history including generalized anxiety disorder, hypertension and hyperlipidemia initially presented to the emergency department for chest pain. Coronary artery bypass graft (CABG) was performed under general anesthesia with Propofol, Fentanyl, Rocuronium and Midazolam. The patient tolerated the procedure well. After the procedure, his stay was complicated by delirium and altered mental status. All reversible causes were investigated and eliminated as likely causes. Later, the patient was sent home after a prolonged hospital stay. Following discharge, he had multiple admissions to the hospital for altered mental status, and his mental status has not reached baseline again. Family has been confident that the patient's psychological and cognitive symptoms began following the CABG.

Conclusion: We present this case to highlight the heightened incidence of postoperative cognitive decline in patients over sixty undergoing major surgery. We also present a comprehensive review of the current literature on the risk factors, clinical manifestations, and pathogenesis of POCD.
\end{abstract}

Key Words: Postoperative cognitive dysfunction, General anesthesia, Alzheimer's disease, Central cholinergic system, Delirium

\section{INTRODUCTION}

Postoperative cognitive dysfunction (POCD) is an under recognized complication of major surgery most commonly observed in geriatric patients. It is associated with significant mortality and morbidity. The disease, also known as postoperative incident dementia and post illness cognitive disorder, is characterized by the development of cognitive impairment within days to weeks of surgery involving anesthesia which may become permanent. While the pathophysiology of POCD remains unclear, the potential for surgical anesthesia to accelerate neurodegeneration is a dubious theme which affects patients as well as specialists, anesthesiologists and outside players in the health care industry. As medical technology continues to improve-along with the survival rates of high risk patients-interventions to optimize visibility and treatment of POCD must improve as well so such patients may survive surgery, critical care and discharge cognitively intact.

\section{CASe PResentation}

A 65-year-old male patient with past medical history of generalized anxiety disorder, coronary artery disease, diverticulosis, dyslipidemia, hypertension, hyperlipidemia, gastroesophageal reflux disease, insomnia, osteoarthritis, paroxys-

*Correspondence: Naveen R Parva; Email: naveenreddy.parva@gmail.com; Address: UPMC Pinnacle, Harrisburg, PA, USA. 
mal atrial fibrillations and peptic ulcer disease presented to the hospital with chest pain and underwent a coronary artery bypass graft $(\mathrm{CABG})$ under general anesthesia with Propofol, Fentanyl, Rocuronium and Midazolam. The patient tolerated the procedure well; however following surgery, the patient's recovery was complicated by delirium and an altered mental status for which he remained in the hospital for 2 months. After this hospital stay, the patient had multiple psychiatric admissions during which the patient's mental status did not returned to baseline.

The patient's most recent psychiatric inpatient admission lasted two months and was complicated by frequent falls (one which resulted in a right intertrochanteric fracture), agitation, dysphagia and multiple other issues. Following this admission, patient was discharged to a rehabilitation center. Later he was admitted to the emergency department by EMS after a homicide attempt on his wife. On initial evaluation, the patient demonstrated sustained eye contact with decreased blinking and mild grimacing. His speech was spontaneous, normal rate, monotone, repetitive and echolalic at times. His thought process was perseverative and occasionally goal directed when asked closed-ended questions, though the patient answered, "I don't know" often. The patient's Mini Mental Status Examination (MMSE) score was $16 / 30$, blood pressure $140 / 82 \mathrm{mmHg}$, pulse 83 beats per minute. Initial labs showed Thyroid stimulating hormone (TSH) level 1.33 milli-international units per liter, Vitamin B12 - 296 pg/ml, Reactive Plasma Reagin was non-reactive. EEG showed no seizure activity and CT scan showed chronic ischemic changes. The differential diagnoses of psychosis and vascular dementia with behavioral changes was considered. The patient was started on high doses of Quetiapine $200 \mathrm{mg}$, Mirtazapine $30 \mathrm{mg}$ qHS, Oxcarbamazepine $450 \mathrm{mg}$, Propranolol $10 \mathrm{mg}$ and Lorazepam $2 \mathrm{mg}$ on multiple admissions. As per family, the patient was fully functional and working as a full-time maintenance staff member who recently retired from pastoral work. Both the patient's family and surgical team believe his mental status changes were a direct result of the $\mathrm{CABG}$ procedure. He was diagnosed with POCD. The patient's mental status has never returned to baseline after $\mathrm{CABG}$ procedure, and he has since been admitted to a rehabilitation center.

\section{Discussion}

The clinical identification of POCD is controversial as it currently does not have an officially accepted diagnostic criteria entry in the Diagnostic and Statistical Manual of Mental Disorders, 5th Edition (DSM-V), nor does it have an International Classification of Disease Code (ICD-10). ${ }^{[1]}$ Moreover, difficulty detecting POCD when it is comorbid with other delirium/dementia related disorders further complicates identification of postoperative decline. As a rule, however, in parsing the differences between the delirium, POCD and dementia, one can identify the type of cognitive disturbance afoot by attention to onset and duration of symptoms after the stressor which could be anesthesia, surgery and/or intensive care. Delirium typically functions on a timeline of days following the stressor; POCD, weeks; and dementia, months. $^{[2]}$

Delirium refers to an acute, fluctuant state of disorientation, decreased awareness and attention span, and confusion, which may also be accompanied by hallucinations. Conversely, a patient with POCD is typically oriented; however, he demonstrates, likely through neurocognitive tests, a significantly poorer performance than expected in one or more cognitive domains following surgery. ${ }^{[3]}$ Dementia, now known in the DSM-V less specifically as major neurocognitive disorder, is defined as a significant, fluctuant cognitive decline from previous performance in the learning and memory, language comprehension and use, executive function, attention, motor coordination and social cognition of the affected individual. The disorder must progress over a period of months at the least to be diagnosed.

\subsection{Prevalence and diagnosis}

Because there are no standard conventions for defining the characteristics of POCD, reported prevalence varies widely between studies; the lack of consistency between the decisions one team of investigators and another make regarding their definition of POCD, the timing of pre and postoperative cognitive testing and which assessments they use to determine the depth of cognitive decline, make determining a true rate of occurrence near impossible. ${ }^{[4]}$ In a study conducted in the first International Study of Postoperative Cognitive Dysfunction (ISPOCD1), 1998, which recruited 947 patients over 60 years and 321 controls in thirteen hospitals spread across the United States and eight European states, POCD was diagnosed in $25.8 \%$ of patients one week following major orthopedic, abdominal or thoracic surgery and in $9.9 \%$ of the same group after three months. ${ }^{[5]}$ In a meta-analysis of studies focusing on the prevalence of POCD in patients following cardiac surgery, at a few weeks after surgery, incidence of POCD was between $30 \%$ and $80 \%$ of patients, and at the time of second testing-3-6 months following surgery-incidence had fallen to $10 \%-60 \% .^{[4]}$

Despite its lack of formal definition, the clinical presentation of POCD has been described in similar ways in recent literature; symptoms generally include: decline in language comprehension, comorbidity with major depressive disorder, impaired executive functioning, memory loss, psychomotor 
agitation, loss of fine-motor coordination, reduced ability to concentrate, and impaired visual memory and abstraction. $^{[6,7]}$ Cognitive status changes are typically temporary, however mental decline can become permanent in some patients (persistent POCD). ${ }^{[8]}$ POCD is generally identified either via a discrepancy between pre and postoperative neurocognitive inventories such as the widely used Mini Mental Status Exam (MMSE), or in the absence of a preoperative baseline score, unexpectedly poor scores accompanied by persistent change in mental status. ${ }^{[1]}$ Generally, such changes in mental status are reported by family members of the patient, colleagues and occasionally, the patient her/himself. ${ }^{[4]}$ Although postoperative delirium is transient in $90 \%$ of the cases, it cannot be considered benign as it can trigger a series of events that adds up leading to increased dependence, death in about $10 \%$ of the cases. ${ }^{[9]}$ The more severe cases culminate into POCD, which can produce catastrophic cognitive impairment, with increased mortality. This augments the risk of prematurely leaving work, and added dependence on social welfare. ${ }^{[2]}$ Because POCD affects multiple mental domains, detection of POCD typically requires a battery of neurocognitive tests, and in a clinical setting, preoperative baseline inventories that indicate $\mathrm{a} \geq 2$ standard deviation difference in pre and postoperative performance indicate POCD. ${ }^{[2]}$ For delirium, the Confusion Assessment Method (CAM) is commonly used. ${ }^{[2]}$ It is particularly utilized in those receiving ventilator support. It incorporates a fourpoint evaluation (acute change or fluctuating course of mental status, inattention, altered level of consciousness and disorganized thinking) and has a high affectability (93\%-100\%) and a specificity (89\%-100\%). The test is likewise straightforward, can be performed by all ICU staff, and takes < 1 min to finish. Another option is the Intensive Care Delirium Screening Checklist. For assessing cognition, accessible nowadays are a progression of electronic tests known as Cogstate battery of tests. To test for dementia particularly in a patient with prior psychological imperfection, the Mini Mental Status Exam (MMSE) or MoCA (Montreal Cognitive Assessment) or Mini-cog can be utilized. ${ }^{[2]}$

\subsection{Pathophysiology}

The pathophysiology of POCD has been theorized to closely resemble the chemical action of both anesthesia and Alzheimer's disease (AD). Anesthetics bind to acetylcholine receptors, causing a delay in regaining full attention, concentration and cognitive ability and a decline in memory, pain, voluntary movements and level of consciousness. ${ }^{[10]}$ Most routine anesthetics, like isoflurane, desflurane and propofol demonstrate chemical action, via action on the cholinergic system, which influences the appearance of known hallmarks of Alzheimer's disease: abnormal processing of

Published by Sciedu Press the $\mathrm{A} \beta$ precursor protein (APP), increased $\mathrm{A} \beta$ peptide production and aggregation of $\mathrm{A} \beta$ proteins into $\beta$-sheets (from $\alpha$-helices). ${ }^{[7,10,17]}$ Additionally, especially high doses of propofol have been linked to increased risk of neurological decline following cardiac surgery. ${ }^{[12]}$ While general anesthesia, relative to regional anesthesia, has been associated with elevated risk of transient POCD, this difference does not appear to extend beyond the first week following surgery at a significant rate. ${ }^{[13]}$

Another proposed mechanism of POCD involves the role of immune response to surgery. This theory is supported mostly with preclinical evidence through animal experiments. Terrando et al. showed that a peripheral surgical procedure in mice activates the inflammatory TNF $\alpha / \mathrm{TNF} \mathrm{B}$ signal cascades, leading to the release of cytokines that impair the integrity of the blood-brain barrier. ${ }^{[14]}$ The compromised blood brain barrier allows for macrophages to migrate easily into the hippocampus leading to memory impairment. If this mechanism is inhibited by anti-inflammatory cholinergic signal cascades, it prevents proinflammatory cytokine secretion leading to unimpaired cognitive function.

Finally, some researchers theorize that the invasiveness of surgery itself is the culprit behind POCD. Mediators of the acute phase response, including cytokines and heat shock proteins, have well-described effects on behavior and learning. Reactive oxygen species and oxidative stress, as seen in severe sepsis, are also associated with decreased cognitive function. ${ }^{[10]}$

\subsection{Health outcomes in individuals with POCD}

In addition to a need to direct attention toward a growing older adult population undergoing surgery with general anesthesia, negative health outcomes linked to cognitive decline during hospitalization should encourage physicians and researchers to screen more effectively and frequently for POCD with the ultimate goal of prevention. ${ }^{[14]}$ Cognitive decline during hospitalization following surgery is linked to greater mortality risk (HR $=1.63,95 \%$ CI: $1.11-2.38),{ }^{[14]}$ increased functional recovery time, and longer hospitalization. ${ }^{[1,4]}$ Social outcomes include inability to perform at job, premature departure from the workforce and resultant financial dependence on social welfare. ${ }^{[2]}$ In the interest of dynamic screening, it is important to emphasize that delirium, POCD and dementia are not unrelated disorders and that the presence of one pre, intra or postoperatively should put medical staff on alert for others; patients who present with delirium immediately after surgery of POCD fare worse than their peers in terms of development of POCD. These patients were at increased risk of poor performance on neuropsychological testing odds ratio for one week: (95\% CI):16.7 (8.7-32.1) and 
for one month: $12.3(7.5-20.0) .{ }^{[12]}$ Most concerning however, is the elevated risk of death in the year following discharge from the hospital with POCD; ${ }^{[14]}$ around $70 \%$ of patients diagnosed with POCD die within five years following surgery as opposed to the $35 \%$ not diagnosed. ${ }^{[7]}$

\subsection{Demographic predictors}

The relationship between all proposed risk factors for POCD is not yet clearly understood, however, one factor most investigators agree upon is the association of increasing age with higher rates of POCD. ${ }^{[3,5,14]}$ The natural neurological changes that accompany aging: decrease in brain-mass, blood flow, permeability of blood-brain barrier and consumption of oxygen in the brain, contribute to the increase in rates of POCD in adults over $65 .{ }^{[15]}$ Additionally, older patients experience a decrease in number and efficiency of acetylcholine receptors, which have been theorized to contribute greatly to the pathogenesis of various types of dementia. ${ }^{[10,16-18]}$ Low education level is also commonly cited as a demographic predictor of postoperative cognitive decline. ${ }^{[5,7]}$ Details surrounding the possible reasons for this association range from reference to socioeconomic disadvantage to "cognitive reserve"-a hypothetical measure of resistance to cognitive decline following brain injury which includes: professional success, years of education and scoring on tests of general knowledge. Evidence currently suggests that cognitive reserve may be protective to $\operatorname{POCD},{ }^{[3]}$ while our literature search has revealed no further socioeconomic predictors of POCD beyond little education.

\subsection{Independent predictors}

Most research conducted on the appearance and character of POCD has been done with regard to its most widely known risk factor: cardiac surgery-most specifically, coronary artery bypass grafting $(\mathrm{CABG}){ }^{[4,7,10]}$ The prevalence of POCD following major cardiac surgery is as high as $70 \%$ $90 \%$, making it a suspected causative factor for POCD, and within cardiac surgeries, $\mathrm{CABG}$ is also considered an independent risk factor in itself. ${ }^{[7]}$ The exact mechanism underpinning the relationship between cardiac surgery and the development of POCD is unknown, however pre-existing heart and vascular disease, especially coronary atherosclerosis and hypertension, are factors that have been found to elevate the risk of POCD. ${ }^{[5,14]}$ The increased prevalence of POCD in cardiac patients postoperatively has also been tentatively explained by microembolic events associated with the use of cardiovascular bypass pump during surgery. ${ }^{[4]}$ These hypotheses, however, are not mutually exclusive are both warrant further, comparative investigation. Future investigation might further observe the relationship between pre-existing cardiac and vascular illness, microembolic action due to the cardiovascular bypass pump and the development of POCD.

Research into shared biological pathways between POCD and $\mathrm{AD}$ indicates that the type of neuronal injury implicated in POCD is similar to the pathogenetic events underpinning the progression of AD. ${ }^{[10,17]}$ However, investigation concerning POCD interaction with vascular dementia, dementia with Lewy Bodies (DLB) and Parkinson's remains sparse, despite evidence that both vascular disease and microembolic action during surgery have been implicated as possible causes of POCD. ${ }^{[4,5,14]}$ Additional psychological risk factors for the development of postoperative cognitive decline come in the form of patient mood; patients with preoperative anxiety and depression, with a depressed baseline mood are at increased risk. ${ }^{[14]}$ Pre and postoperative usage of benzodiazepines for anxiety and depression has been associated with higher incidence of POCD and delirium. ${ }^{[2,13,15]}$ Interestingly, both alcohol avoidance and abuse have been suggested as predisposing factors for POCD. ${ }^{[3,14]}$

Table 1. Postoperative cognitive dysfunction risk inventory

\begin{tabular}{l}
\hline Factor \\
\hline Cardiac surgery; CABG \\
Pre-existing dementia or MCI \\
Age over 63 \\
H/o major orthopedic surgery \\
H/o other invasive surgery \\
H/o depression \\
H/o benzodiazepine use \\
H/o postoperative delirium \\
H/o institutionalization \\
Atherosclerosis \\
Stroke; chronic ischemic changes \\
Hypertension \\
Little education (no post-secondary education) \\
\hline
\end{tabular}

\subsection{Clinical suggestions}

While current literature does not represent a consensus on the mechanics of POCD, we have noted and explained possible risk factors. From these we offer the following suggestions for clinical practice regarding preventing and alleviating effects of postoperative cognitive dysfunction:

(1) Discuss POCD as a possible complication of major cardiac, especially coronary artery bypass grafting and/or orthopedic surgery with the patient and family. We suggest making POCD a point of conversation if the patient presents with any of the conditions listed on the risk factor table below before surgery (see Table 1).

(2) We recommend a test battery including inventories 
commonly used to detect dementia and/or mild cognitive impairment for tracking patients' baseline cognitive function prior to surgery. Following surgery, we suggest performing the same tests one week, and one month afterward while accounting for patient familiarity with examinations. Immediately following surgery, we recommend administering the CAM-ICU test as per the demonstrated connection between incidence of postoperative delirium and POCD.

(3) Pre and Intraoperative considerations include:

- Explaining patients' risk status as per table to entire surgical team before scheduled surgery time.

- Usage of minimal depth of anesthesia possible and avoidance of high doses of propofol and sevoflurane.

(4) Postoperative considerations include:

- Post-operative pain management strategies which aggressively minimize inflammation.

- Avoidance of pre (if possible) and post-operative benzodiazepine use.

- Follow up with patient and family after surgery regarding any changes in mental status.

\section{Conclusion}

POCD is an under perceived complication of surgery which can cause irreversible damage to cognitive function. In this review, we have offered clinical and preclinical evidence for both risk factors and the possible pathogeneses of POCD. In recognizing that a patient is at preoperative high risk, clinicians can initiate collaboration with the patient, her/his family and the surgical team to explain the risk of POCD, take preventive actions and decrease depth of possible cognitive impairment. Considering our patient's presentation before his eventual diagnosis of POCD, we recognize the patient demonstrated most known risk factors for POCD. We believe that bringing a greater depth and breadth of knowledge of POCD into the initial process of determining patient eligibility for surgery can decrease incidence and severity of POCD. However, further studies to stratify risk factors, determine pathogenesis and clinically test patient prognoses with treatments which aggressively minimize inflammation and opioid pain management are warranted.

\section{CONFLICTS OF INTEREST Disclosure}

The authors have declared no conflicts of interest.

\section{REFERENCES}

[1] Arora SS, Gooch JL, Garcia PA. Postoperative cognitive dysfunction, Alzheimer's disease, and anesthesia. Int J Neurosci. 2014; 41(4): 236-42. PMid:23931049 https ://doi .org/10.3109/00207454 .2013 .833919

[2] Lloyd DG, Ma D, Vizcaychipi MP. Cognitive decline after anaesthesia and critical care. Continuing Education in Anaesthesia, Critical Care \& Pain. 2012; 12 (3): 105-9. https://doi.org/10.1093/ bjaceaccp/mks004

[3] Tsai TL, Sands LP, Leung JM. An Update on Postoperative Cognitive Dysfunction. Advances in anesthesia. 2010; 28(1): 26984. PMid:21151735 https://doi.org/10.1016/j.aan.2010 .09 .003

[4] Wang F. Postoperative Cognitive Dysfunction: Current Developments in Mechanism and Prevention. Medical Science Monitor. 2014; 20: 1908-12. PMid:25306127 https://doi.org/10.12659/MSM .892485

[5] Moller J, Cluitmans P, Rasmussen L, et al. Long-term postoperative cognitive dysfunction in the elderly: ISPOCD1 study. The Lancet. 1998; 351(9106): 857-61. https://doi .org/10.1016/S0140-6 736 (97) 07382-0

[6] $\mathrm{Hu} \mathrm{Z}$, Ou Y, Duan K, et al. Inflammation: a bridge between postoperative cognitive dysfunction and Alzheimer's disease. Medical Hypotheses. 2010; 74(4): 722-4. PMid:19944539 https : //doi .or $\mathrm{g} / 10 \cdot 1016 / \mathrm{j}$. mehy $\cdot 2009 \cdot 10 \cdot 040$

[7] Fodale V, Santamaria LB, Schifilliti D, et al. Anaesthetics and postoperative cognitive dysfunction: a pathological mechanism mimicking Alzheimer's disease. Journal of the Association of Anes- thetists of Great Britain and Ireland. 2010; 65(4): 388-95. https : //doi.org/10.1111/j.1365-2044.2010.06244.x

[8] Mashour GA, Woodrum DT, Avidan MS. Neurological complications of surgery and anaesthesia. British Journal of Anaesthesia. 2014; 114(2): 194-203. PMid:25204699 https ://doi.org/10.1 093/bja/aeu296

[9] Sheehan B. Assessment Scales in Dementia. Therapeutic Advances in Neurological Disorders. 2012; 5(6): 349-58. PMid:23139705 https://doi.org/10.1177/1756285612455733

[10] Fodale V, Quattrone D, Trecroci C, et al. Alzheimer's disease and anesthesia: implications for the central cholinergic system. British Journal of Anesthesia. 2006; 97(4): 445-52. PMid:16950812 https://doi.org/10.1093/bja/ael233

[11] Xie Z, Tanzi RE. Alzheimer's disease and post-operative cognitive dysfunction. Experimental Gerontology. 2006; 41: 346-59. PMid:16564662 https://doi .org/10.1016/j . exger . 2006.0 1.014

[12] Chan MTV, Cheng BCP, Lee TMC, et al. BIS-guided Anesthesia Decreases Postoperative Delirium and Cognitive Decline. Journal of Neurosurgical Anasthesiology. 2013; 25(1): 33-42. PMid:23027226 https://doi.org/10.1097/ANA.0b013e3182712f ba

[13] Zywiel MG, Prabhu A, Perruccio AV, et al. The Influence of Anesthesia and Pain Management on Cognitive Dysfunction After Joint Arthroplasty: A Systematic Review. Clinical Orthopaedics and Related Research. 2013; 472(5): 1453-66. PMid:24186470 https: //doi.org/10.1007/s11999-013-3363-2

[14] Rundshagen I. Postoperative Cognitive Dysfunction. Deutsches Ärzteblatt International. 2014; 111(8): 119-25. PMid:24622758 
[15] Rana MV, Bonasera LK, Bordelon GJ. Pharmacologic Considerations of Anesthetic Agents in Geriatric Patients. Anesthesiology Clinics. 2017; 35(2): 259-71. PMid:28526147 https://doi.org/10.101 6/j.anclin.2017.01.011

[16] Lazic K, Petrovic J, Ciric J, et al. Impact of anesthetic regimen on the respiratory pattern, EEG microstructure and sleep in the rat model of cholinergic Parkinson's disease neuropathology. Neuroscience. 2015; 304: 1-13. PMid:26186897 https://doi.org/10.1016/j.neur oscience. 2015.07 .020
[17] Everitt BJ, Robbins TW. Central Cholinergic Systems and Cognition. Annual Review of Psychology. 1997; 48: 649-84. PMid:9046571 https://doi.org/10.1146/annurev.psych.48.1.649

[18] Praticò C, Quattrone D, Lucanto T, et al. Drugs of anesthesia acting on central cholinergic system may cause post-operative cognitive dysfunction and delirium. Medical Hypotheses. 2005; 65(5): 972-82. PMid:16043305 https://doi.org/10.1016/j.mehy .2005.05 .037 